\title{
The Effects of Hybrid Fertilizer on Plant Life
}

\author{
Eva Saramya \\ Forsyth Country Day School, 5501 Shallowford Rd, Lewisville, North Carolina, USA; esaramya@gmail.com
}

ABSTRACT: Current research suggests that roughly $50 \%$ of all food that is produced around the world is wasted. Finding a sustainable solution to the global compost waste issue was the primary motivation for this study. This analysis encompasses the first-ever quantitative data sought for "Soil Sauce", an organic-compost based liquid fertilizer. To understand the impact(s) of fertilizer on the efficacy of plant growth, a lab analysis - Procedure I - along with a fertilizer test - Procedure II - were conducted to gain more insight into the product's ability to aid in plant growth and reduce food waste. The lab analysis involved observing the properties of soil sauce under a microscope using the Gram's sample staining method. Procedure II involved applying quantities ranging from 2-6 drops of soil sauce into distinct plant groups to test product efficiency.

A basic understanding of fertilizer, what it possesses, differences between commercial and compost-based fertilizers, and fundamental knowledge of bacterial shapes were needed as a foundation to thoroughly comprehend the different parts of this study thoroughly. The main conclusions from this experiment were that "Soil Sauce" is, in fact, extremely beneficial for plant growth. The results showed that the plant group with the most drops of "Soil Sauce" grew significantly better compared to its counterparts, the plant groups with less drops of soil sauce and the control group.

KEYWORDS: Earth and Environmental Sciences; Hybrid Fertilizer; Soil Sauce; Bacterial Ecology; Environmental Effects on Ecosystems.

\section{- Introduction}

"Soil Sauce" is a hybrid fertilizer made in Pittsburg, Pennsylvania by the Ecotone Renewables Team. The fertilizer is described as "hybrid" due to its unique combination of properties from both compostable and chemicalized fertilizers. The process to make "Soil Sauce" involves taking leftover vegetables and fruits and putting the scraps through a manufacturing process involving an anaerobic digester. Anaerobic digestion is the process in which organic matter is broken down to produce biogas or biofertilizer. This process occurs in the absence of oxygen in a sealed oxygen-free tank known as an anaerobic digester. ${ }^{1}$

Fertilizer is a solution composed of essential elements and nutrients needed to make plants grow rapidly and healthier. Important elements such as nitrogen, phosphorus, potassium, magnesium, sulfur, and calcium are all needed in successful plant growth. Nitrogen (nitrate) strengthens foliage during the critical development stage. Nitrate also contributes to chlorophyll production, responsible for creating the signature green color commonly seen in many plants. Phosphorus aids in root growth and offers protection to plants against harmful environmental stresses and factors. ${ }^{2}$ Potassium helps in water retention, contributes to early plant growth, and protects against insects and diseases. Magnesium aids in chlorophyll production by absorbing sunlight during photosynthesis. Magnesium is also critical to plant organisms for cell division, protein/enzyme formation, cell membrane stabilization, and metabolic functions in the plant. ${ }^{3}$ Sulfur aids in protecting the plant organism from disease and helps with the production of amino acids, enzymes, proteins, and vitamins. Calcium contributes to the growth and development of the cell wall, which is critical as the cell wall is the first line of defense for the plant organism in withstanding harmful diseases. ${ }^{4}$

One of the objectives of this study was to classify where "Soil Sauce" fits in the spectrum of fertilizers. This research was critical in understanding how exactly "Soil Sauce" works. Roughly $50 \%$ of all food that is produced around the world is wasted. ${ }^{5}$ By understanding Soil Sause proper classification, in terms of fertilizer, it distinguished how exactly "Soil Sauce" aids in plant enrichment whilst potentially reducing global waste and improving soil health through utilization of natural, reusable compostable waste. By understanding where "Soil Sauce" fits within fertilizer classification, it can be distinguished how this plant-enriching product can potentially reduce global waste and improve soil health by utilizing natural, reusable, compostable waste. Additionally, we can understand how the use of "Soil Sauce" can contribute to regenerative agriculture and preserve vital soil nutrients.

The spectrum of fertilizers, ranging from compost-based to chemicalized, entails a variety of options to enrich plant life. Fertilizer provides the vital nutrients plants need to regenerate and grow effectively. The creation of fertilizer is to bring back the nutrients the soil loses through the previous plant cycle continuing the food production process effectively. The broad types of fertilizers include mineral rich, nutrient rich, and chemicalized fertilizers. These fertilizers tend to capitalize on specific vital nutrients, such as calcium, phosphorus, magnesium, to promote plant growth. Mineral-based fertilizers transform naturally occurring, geologically derived raw materials into plant nutrients. Conversely, there are organic fertilizers typically comprised from cow manure, left over crop yield, or compost. These fertilizers, unlike their chemicalized 
counterparts, depend on their composition by way of regional resources. Organic fertilizers are ambiguous in terms of consistent nutrient compounds. As resources and crop yields change the organic fertilizer, the makeup adheres to the change as well, providing different nutrient compositions per each batch. This does not take away from its ability to benefit plant growth and proves to be just as effective as its chemicalized counter parts. ${ }^{6}$ Nevertheless, both organic and inorganic nutrients are important to sustainable crop productivity and soil health. ${ }^{7}$

A compost-based fertilizer is composed primarily of compost consisting of natural ingredients such as leftover fruits and vegetable scraps. Compost has notably assisted in plant growth due to its soil-enriching properties. It is associated with improving texture and aiding in creating a healthier environment for plant life due to its composition. Organisms found in compost such as bacteria, fungi, redworms, and dung beetles replenish and provide nutrients to the soil, correlating with successful and healthy plant growth. Commercial fertilizer is mostly, if not entirely, chemical-based. The vitamins and minerals needed are chemically processed and manufactured from raw elements and compounds such as ammonia (NH3). The elements are broken down chemically or manually with machinery to make the process of absorbing the vital nutrients easier for the soil. ${ }^{8}$

The main gap found throughout prior research was the lack of proper classification for what "Soil Sauce" is in terms of fertilizers on the market today. "Soil Sauce" is classified as a chemicalized fertilizer made out of compost; it combines elements from both the compost-based and commercially chemicalized fertilizers, placing the product within its own hybrid classification. Since this category of hybrid fertilizer hasn't been researched in detail, it made distinguishing "Soil Sauce" as a proper fertilizer cumbersome and made finding information on its properties difficult.

Understanding the basic bacterial shapes, arrangements, sizes, and organisms found in many fertilizers is beneficial. It offers reference and a basic framework on what needed to be looked for and observed, especially during Procedure I, to be detailed later in this paper.

The three shapes that were looked at were cocci, spirilla, and bacilli cells. Coccus (single cell) is a round, spherical shaped cell. Spirilla cells (spirillum for single cells) are bacteria with a distinct curve range varying from gently curved to tight corkscrews. Spirilla cells are rigid and capable of movement. Bacilli (bacillus for single cell) are rod-shaped bacteria. ${ }^{9}$ Soil enriching organisms such as gastrotrichs were also looked for and observed.

The objectives of this study were to measure and establish concrete quantitative data for "Soil Sauce" as this fertilizer had no data previously. Objective 1 was to collect data points to see if "Soil Sauce" had the capabilities to accelerate plant growth. Objective 2 involved seeing if "Soil Sauce" could potentially be an alternative needed to minimize global compost waste whilst helping and nourishing the environment.

\section{- Methods}

\section{Measurements (Variables):}

The independent variable was the amount of fertilizer in each batch of mustard plant samples. The dependent variable was how tall the mustard plants grew. The constants included the experimental material, procedures, and the amount of water, sunlight, and soil each plant received.

\section{Design:}

The procedures were critical in analyzing and understanding "Soil Sauce" from all perspectives and finding the information needed to test and prove its efficiency. Procedure I involved evaluation and observation of chemical and bacterial make-ups. Procedure I was also crucial in comparing "Soil Sauce" to fertilizers on the market today for possible correlations. Procedure II directly tested "Soil Sauce" on plant life. Procedure II was vital in testing the efficiency of the fertilizer.

The experimental design involved growing mustard plants and observing growth rate after the addition of Soil Sauce. Thus, the execution of the procedures was critical in data identification. The aim was to take the growth rate into account and gain calculations to support the research hypothesis: the plant group with the most soil sauce will thrive the best. The results and data were then placed in charts and analyzed. Data for each group was reported separately. The data compiled the growth rates of each plant group per day for approx. 1 week. The data helped identify patterns, correlations, and constancies in plant growth. A chart of growth throughout the week, the averages for each day, and a visual data curve were created based on each group.

\section{Procedure I:}

Procedure I involved testing, analyzing, and observing the bacterial and chemical compositions of "Soil Sauce" using the Gram's Staining Method; a process of finding bacteria involving a methodical repetition of staining and washing until a final stained product is achieved. The materials used were microscope slides, matches, alcohol lamps, crystal violet stain, safranin stain, isopropyl alcohol, gram's iodine stain, agar plates, loop, and tryptic soy broth cultures. The alcohol lamps were heated to sterilize the loop after each use to avoid contamination from sample to sample. The heated loop was then put into "Soil Sauce" and then placed onto a labeled agar plate. The samples were then incubated with the broth cultures at $37^{\circ} \mathrm{C}$ to develop over 24 hours. After the incubation period, the samples were subjected to the Gram's Staining method. The sterilized loop was then used to scrape off a part of the incubated sample and was placed on a microscope slide with a drop of water. The water was combined with the scraped sample to create a homogenous mixture. The slide was then placed on and off an open fire, fueled by the alcohol lamps, until the water and excess liquid evaporated. What was left was the evaporated residual sample of "Soil Sauce" on the microscope slide. Crystal violet was then applied onto the cooled sample for 20 seconds. It was rinsed off with water, which was then followed up with gram's iodine for $1 \mathrm{~min}$. The isopropyl alcohol was then used to decolorize the gram's iodine after $1 \mathrm{~min}$. The isopropyl alcohol was then washed off with water and was followed by the safranin for 20 seconds. 
The final wash of water was then used to rinse off the excess stain and the slide was blot dried until no water or liquids were left. Since 5 samples of "Soil Sauce" were taken, this process was repeated 5 times. Finally, each of the 5 samples were put under the microscope to be observed.

\section{Procedure II:}

Procedure II involved actively testing "Soil Sauce" on plant life and recording the data accumulated. The hypothesis "the plants with the most fertilizer will thrive the best" guided this part of the experiment. Some materials used included egg cartons as planters, tray, eyedropper, "Soil Sauce" fertilizer, cups, soil, and mustard seeds. This experiment was set up and split into 4 main groups. The first group was the control group with no fertilizer, labeled and marked with the blue popsicle stick. The second, third, and fourth groups all contained varying amounts of soil sauce. Group 2 had the least amount of fertilizer (1-2 drops) represented by the red popsicle stick. Group 3 contained 3-4 drops of fertilizer, represented by the yellow popsicle stick. Group 4 had the most amount of fertilizer (5-6 drops) and was represented by the green popsicle stick. All groups received equal amounts of soil, water, sunlight, and seeds. The tray was placed near a window for a consistent, adequate source of sunlight. The egg cartons were then placed onto the tray along with the dampened soil within them. The popsicle sticks were then cut and labeled with a color and placed into its allotted group to keep data collection organized. $14 \mathrm{ml}$ (approx. 1 tsp) of seeds were added to each big group (each big group was comprised of four mini-groups) and then covered up with the remaining soil. 14 to $28 \mathrm{ml}$ of water along with the specific amounts of "soil sauce" per group were added every day for 5 days until the experiment concluded. Data was recorded and accumulated through measuring each plant individually, consistently every day until the conclusion of the experiment.

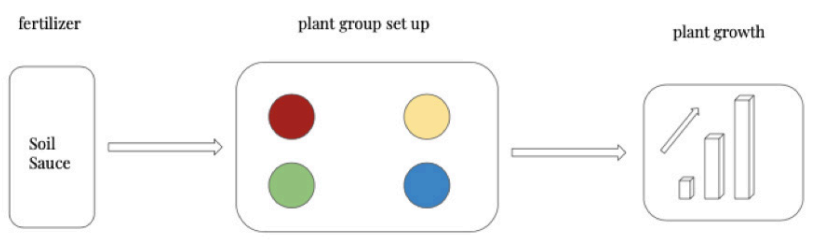

Figure 1: Experiment schematic design. The varying quantities of soil sauce on plant growth are represented through the final factor of growth.

The schematic above outlines how Procedure II was conducted. The varying quantities of soil sauce on plant growth are represented through the final factor of growth.

\section{Results and Discussion}

The samples included bacillus cells while others contained cocci. Some samples contained both cell types while others only had one or the other. None of the samples consisted of spirilla cells. The bacteria range observed depended on the specific sample taken. Each sample had a very different chemical and bacterial composition, thus making "Soil Sauce" an extremely complex and multilayered product. A soil organism known as a gastrotrich was also found among one of the samples. Gastrotrichs are common aquatic detritivores, that help break down complex organic material, found usually in the liquid compost (refer to Appendix for details).
The overall growth average (OGA), took the average of the averages from each day (refer to Table 6), was also determined for all plant groups (refer to the Tables 1-4 \& Figures 2 - 5 below). Table 2 focuses on the plant group data with the least "low" amount of fertilizer (red group). Table 3 contains the data for the yellow group; the group contained the "medium" amount of fertilizer. Table 4 covers the data of the green group; the plants that received the most drops of fertilizer ("high"). The blue group was the control group. No fertilizer was added to this group. All plants (regardless of grouping) received the same amount of sunlight, water and were subjected to the same temperature. The data recorded for all plants was over a 5-day period and was recorded down at the same time every day.

Table 1: Data for red batch samples. Active, consistent growth starts at day 3 and progresses throughout the experiment.

\begin{tabular}{|l|l|l|l|l|}
\hline red sample (1-2 drops of fertilizer) & plant 1 & plant 2 & plant 3 & plant 4 \\
\hline day 1 & $0 \mathrm{~cm}$ & $0 \mathrm{~cm}$ & $0 \mathrm{~cm}$ & $0 \mathrm{~cm}$ \\
\hline day 2 & $0 \mathrm{~cm}$ & $0 \mathrm{~cm}$ & $0 \mathrm{~cm}$ & $0 \mathrm{~cm}$ \\
\hline day 3 & $1 \frac{112 \mathrm{~cm}}{1 / 2 \mathrm{~cm}}$ & $1 \frac{1 / 2 \mathrm{~cm}}{1} \mathrm{~cm}$ \\
\hline day 4 & $13 / 4 \mathrm{~cm}$ & $1 \frac{1 / 2 \mathrm{~cm}}{3 \mathrm{~cm}}$ & $2 \mathrm{~cm}$ \\
\hline day 5 & $2 \mathrm{~cm}$ & $2 \mathrm{~cm}$ & $3 \frac{1 / 2 \mathrm{~cm}}{21 / 2 \mathrm{~cm}}$ & \\
\hline
\end{tabular}

RED PLANT SAMPLE

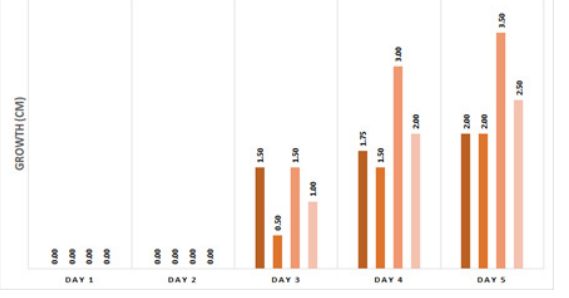

Figure 2: Data chart for red batch samples. The data in day 4 mirrors that of day 5 showing the consistent upward trend in plant growth.

Table 2: Data for yellow batch samples. All plants started growing at day 4 and continued to show promising growth onwards.

\begin{tabular}{|c|c|c|c|c|}
\hline Yellow sample (3-4 drops of fertilizer) & plant 1 & plant 2 & plant 3 & plant 4 \\
\hline day 1 & $0 \mathrm{~cm}$ & $0 \mathrm{~cm}$ & $0 \mathrm{~cm}$ & $0 \mathrm{~cm}$ \\
\hline day 2 & $0 \mathrm{~cm}$ & $0 \mathrm{~cm}$ & $0 \mathrm{~cm}$ & $0 \mathrm{~cm}$ \\
\hline day 3 & $0 \mathrm{~cm}$ & $1 \frac{1}{2} \mathrm{~cm}$ & $0 \mathrm{~cm}$ & $1 \mathrm{~cm}$ \\
\hline day 4 & $2 \mathrm{~cm}$ & $2 \mathrm{~cm}$ & $1 \mathrm{~cm}$ & $2 \mathrm{~cm}$ \\
\hline day 5 & $2 \frac{1}{2} \mathrm{~cm}$ & $23 / 4 \mathrm{~cm}$ & $1 \frac{1}{2} \mathrm{~cm}$ & $2 \frac{1}{2} \mathrm{~cm}$ \\
\hline
\end{tabular}




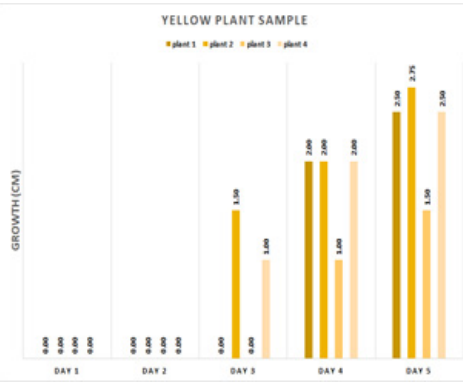

Figure 3: Data chart for yellow batch samples. The data in days 4 and 5 is mostly consistent within the plants with 3 out of 4 plants having close growth rates

Table 3: Data for green batch samples. All plants grew consistently from day 2.

\begin{tabular}{|l|l|l|l|l|}
\hline Green sample (5-6 drops of fertilizer) & plant 1 & plant 2 & plant 3 & plant 4 \\
\hline day 1 & $0 \mathrm{~cm}$ & $0 \mathrm{~cm}$ & $0 \mathrm{~cm}$ & $0 \mathrm{~cm}$ \\
\hline day 2 & $1 \frac{1 / 2 \mathrm{~cm}}{1 \frac{1}{2} \mathrm{~cm}}$ & $1 \mathrm{~cm}$ & $3 \mathrm{~cm}$ \\
\hline day 3 & $3 \mathrm{~cm}$ & $2 \mathrm{~cm}$ & $3 \mathrm{~cm}$ & $3 \mathrm{~cm}$ \\
\hline day 4 & $4 \mathrm{~cm}$ & $3 \mathrm{~cm}$ & $4 \frac{1 / 2}{\mathrm{~cm}}$ & $5 \mathrm{~cm}$ \\
\hline day 5 & $5 \mathrm{~cm}$ & $4 \mathrm{~cm}$ & $5 \frac{1 / 2 \mathrm{~cm}}{51 / 2 \mathrm{~cm}}$ & \\
\hline
\end{tabular}

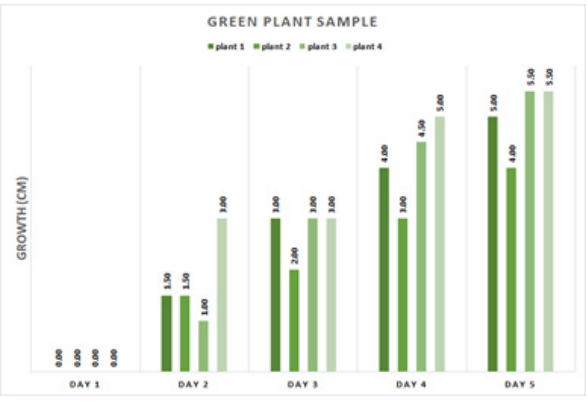

Figure 4: Data chart for green batch samples. Growth data appears in a steady upward progression from day 2 onwards.

Table 4: Data for blue batch samples. Plants start growing from day 2.

\begin{tabular}{|l|l|l|l|l|}
\hline Blue sample (control group) & plant 1 & plant 2 & plant 3 & plant 4 \\
\hline day 1 & $0 \mathrm{~cm}$ & $0 \mathrm{~cm}$ & $0 \mathrm{~cm}$ & $0 \mathrm{~cm}$ \\
\hline day 2 & $1 / 2 \mathrm{~cm}$ & $1 / 2 \mathrm{~cm}$ & $1 \mathrm{~cm}$ & $1 / 2 \mathrm{~cm}$ \\
\hline day 3 & $1 \frac{1 / 2 \mathrm{~cm}}{2 \mathrm{~cm}}$ & $3 \mathrm{~cm}$ & $1 \frac{1 / 2 \mathrm{~cm}}{}$ \\
\hline day 4 & $3 \frac{1 / 2 \mathrm{~cm}}{31 / 2 \mathrm{~cm}}$ & $3 \mathrm{~cm}$ & $4 \mathrm{~cm}$ \\
\hline day 5 & $3 \frac{3}{4} \mathrm{~cm}$ & $4 \mathrm{~cm}$ & $4 \mathrm{~cm}$ & $4 \frac{1 / 2}{\mathrm{~cm}}$ \\
\hline
\end{tabular}

BLUE PLANT SAMPLE

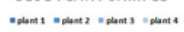

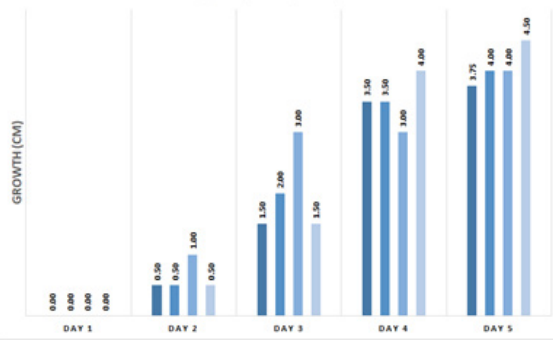

Figure 5: Data chart for blue batch samples. Plant Data has a significant jump from day 2 to 3 . From Day 3 onwards data has a steady, consistent upward trend.

Table 6: OGA Chart. The purpose of the chart is to show the overall average of growth per each plant group. The data present supports that the plant group that received the most fertilizer (group green) grew exponentially well overall compared to the other plant groups recorded.

\begin{tabular}{|l|l|}
\hline Plant Group Name & Overall Growth Average (OGA) \\
\hline red & $1.1375 \mathrm{~cm}$ \\
\hline yellow & $0.9375 \mathrm{~cm}$ \\
\hline green & $2.725 \mathrm{~cm}$ \\
\hline blue & $1.9875 \mathrm{~cm}$ \\
\hline
\end{tabular}

Table 6a: OGA data probability distribution.

\begin{tabular}{|l|r|r|}
\hline & \multicolumn{1}{|l|}{$\begin{array}{l}\text { Plant } \\
\text { Growth }\end{array}$} & Probability Distribution \\
\hline Yellow & 0.9375 & 0.3173 \\
\hline Red & 1.1375 & 0.4114 \\
\hline Blue & 1.9875 & 0.5152 \\
\hline Green & 2.725 & 0.1977 \\
\hline
\end{tabular}

\begin{tabular}{|l|r|}
\hline Mean & 1.70 \\
\hline Standard Deviation & 0.71 \\
\hline
\end{tabular}

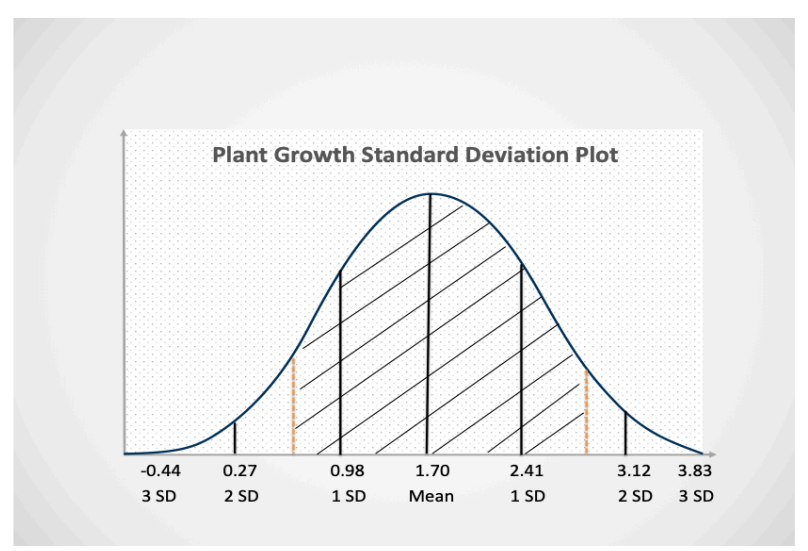

Figure 6: Standard deviation plot.

The plot displays the average standard deviation for overall plant growth represented by the calculated overall growth average (OGA). The chart shows that about $90 \%$ of the study 
data falls within 2 standard deviations from the mean, represented between the two orange lines. This shows that the sample data used lies within the statistically significant range.

\section{Observations:}

Some observations to note from Procedure II were that all the plants that received "Soil Sauce" appeared to look healthier, with a darker, richer green pigment, especially compared to the control group. The control group plants look quite malnourished, underdeveloped, and were lacking in the rich healthy color, instead a pale whitish green, compared to their fertilized counterparts. From this alone, it can be concluded that "Soil Sauce" not only accelerates plant growth but also promotes healthier, well-nourished plant life.

\section{- Discussion}

From what was analyzed, "Soil Sauce" does have all the elements and resources needed to potentially be a successful fertilizer. The efficiency in plant growth throughout the experiment supports this conclusion. Testing different fertilizers on a common plant group and comparing it with "Soil Sauce's" efficacy rates could provide more insight and better understanding of soil sauces' abilities, especially when compared to its commercial and organic counterparts. Note that the quantities of soil sauce put into the red, yellow and green plant groups were relatively minimal. Experimenting with different quantities of "Soil Sauce" could lead in finding the optimal amount for the most substantial plant growth. The experiment did lack in variety because only one plant type was tested.Testing "Soil Sauce" on plants in addition to mustard seed could be integral in understanding the range of "Soil Sauce's" benefits and limitations. Manipulating the time of the conducted experiment could also observe if "Soil Sauce" is consistently effective long term. Overall, the efficacy of compost-based fertilizer is very promising and could potentially revolutionize the agricultural industry.

\section{- Conclusion}

In conclusion, both procedures suggest that the hybrid fertilizer "Soil Sauce" is promising for excellent plant growth. The most conclusive and promising data included the green group - the plant group that received the most fertilizer - which generated healthy plant life exponentially well at a consistent rate compared to the other groups tested. The groups fertilized at lower amounts did show growth, but not at the rate the green groups growth. Practical applications with the data collected from this experiment could revolutionize the agricultural industry greatly and in a positive way, especially with the high and popular demand for organic food. From a financial standpoint, if "Soil Sauce" was in retail, more farmers could grow their crops organically, leading to more income for farmers and customer satisfaction for consumers. This research could potentially help the environment by limiting chemical runoff into water areas, eliminating the risk of water contamination, and providing safety assurance for marine life. ${ }^{10} \mathrm{On}$ a smaller note "Soil Sauce" could also help the avid home gardeners with their plants. Since "Soil Sauce" is made up of liquid compost the waste from compostable foods would also go down tremendously. According to Ecotone Renewables "Globally, 30-40 \% of all food that is produced is wasted". ${ }^{11}$ This experiment suggests that "Soil Sauce" could potentially reduce global waste

by utilizing natural, reusable, compostable surplus. The uses for this multipurpose fertilizer are endless and could change the world for the better one day.

\section{Acknowledgements}

I wish to thank both Dr. Peter Kish and Dr. Gabriella Dee for all their help and support. Dr. Kish for providing the lab facility to guide me through the lab procedures and Dr. Dee for providing access to soil sauce. I would also like to thank Dr. Saravana "Samy" Govindasamy for help in editing and polishing the paper.

\section{References}

1. Interdirect. What is anaerobic digestion? http://www.biogen.co.uk/ Anaerobic-Digestion/What-is-Anaerobic-Digestion (accessed Feb $28,2021)$.

2. https://www.holganix.com/blog/why-your-plants-needphosphorus-advantages-and-disadvantage (accessed Feb 28, 2021).

3. https://www.gardeningknowhow.com/garden-how-to/ soil-fertilizers/fixing-magnesium-deficiency (accessed Feb 28, 2021).

4. Ersek, K. The 6 essential nutrients for healthy plants https://www. holganix.com/blog/the-6-essential-nutrients-for-healthy-plants (accessed Feb 28, 2021).

5. Smithers, R. Almost Half of the World's Food Thrown Away, Report Finds. The guardian. January 10, 2013.

6. Types of fertilizer - fertilizers Europe https://www.fertilizerseurope. com/fertilizers-in-europe/types-of-fertilizer (accessed Feb 28, 2021).

7. Mahmood, F.; Khan, I.; Ashraf, U.; Shahzad, T.; Hussain, S.; Shahid, M.; Abid, M.; Ullah, S. Effects of Organic and Inorganic Manures on Maize and Their Residual Impact on Soil PhysicoChemical Properties. J. soil sci. plant nutr. 2017, 17 (ahead), 0-0.

8. How fertilizer is made - material, production process, making, history, used, components, composition, product http://www. madehow.com/Volume-3/Fertilizer.html (accessed Feb 28, 2021).

9. Aryal, S. Different Size, Shape and Arrangement of Bacterial Cells https://microbiologyinfo.com/different-size-shapeand-arrangement-of-bacterial-cells (accessed Feb 28, 2021).

10.Espinoza, R. Chemical waste that impact on Aquatic Life or Water Quality https://blog.idrenvironmental.com/chemicalwaste-that-impact-on-aquatic-life-or-water-quality (accessed Feb 28, 2021).

11.About https://ecotonerenewables.com/About (accessed Feb 28, 2021).

\section{Author}

Eva Saramya is a senior in high school and is interested in the field of medical/health sciences. She is planning to major in microbiology, immunology and/or environmental sciences. She's loved gardening from a young age and looking into the microbial aspects of plant life further has amplified her love not only for ecology but for microbiology as well.

\section{Appendix}

\section{A. BACTERIAL SAMPLES (PROCEDURE I)}

\#1

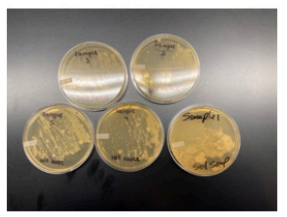

\#2

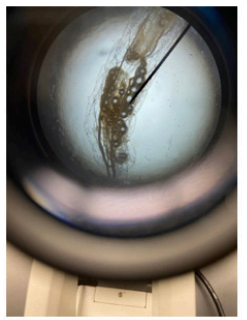




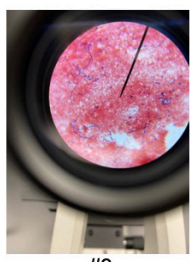

\#3

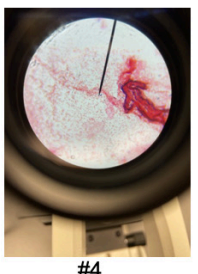

\#4

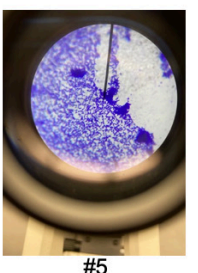

\#5

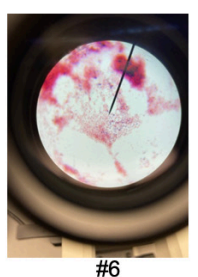

\#6

\#1: shows the incubated soil sauce samples

\#2: gastrotrichs found in drop sample of soil sauce under microscope (no incubation or gram's staining process)

\#3 - 6: taken from incubated samples of soil sauce, put through grams' staining method and observed under microscope. All pictures contain cocci (red) and bacilli cells (blue). Through this process it was concluded that the composition of soil sauce remains to be complex and somewhat ambiguous. Within one batch itself that composition varied greatly from each sample.

Note: All samples were collected from 1 batch of soil sauce.

B: PLANT GROUP SET UP (PROCEDURE II)

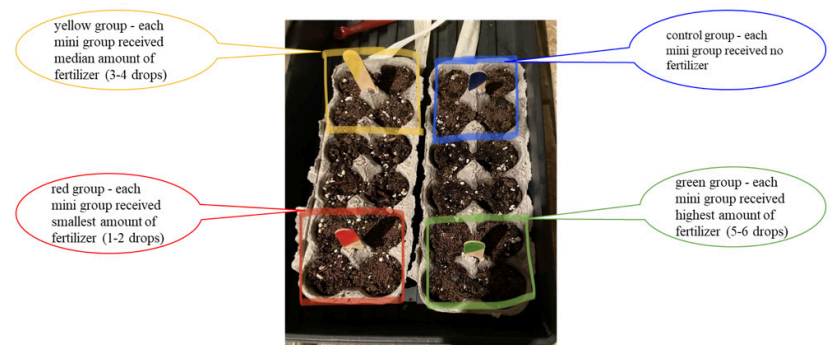

- Each highlighted big group is comprised of 4 mini-groups

- Control group (top right, highlighted with blue square) each mini group received no fertilizer

- Red group (bottom left, highlighted with red square) each mini group received smallest amount of fertilizer (1-2 drops)

- Yellow group (top left, highlighted with yellow square) - each mini group received median amount of fertilizer (3-4 drops)

- Green group (bottom right, highlighted with green square) - each mini group received the highest amount of fertilizer (5-6 drops)

c: ECOTONE RENEWABLES TEAM

(GROUP RESPONSIBLE FOR CREATING “SOILSAUCE”)

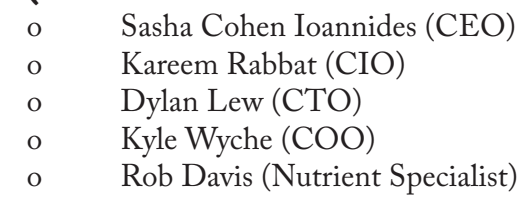

